\title{
Calculation of linear technological dimensions with adaptation
}

\author{
Vasily Masyagin ${ }^{1, *}$, Ruslan Bazhenov ${ }^{2}$, and Roman Artyukh ${ }^{1}$ \\ ${ }^{1}$ Omsk State Technical University, Mira 11, Omsk, 644050, Russia \\ ${ }^{2}$ Sholom-Aleikhem Priamursky State University, Shirokaya 70a, Birobidzhan, 679015, Russia
}

\begin{abstract}
Technological dimensional calculations are a necessary stage of engineering process and allow to determine machining allowance. The problem is that, when processing hard-to-treat materials on NC machines, it is necessary to provide optimal processing conditions for each workpiece based on reliable information on allowances at each operation. However, according to the existing methodology for calculation of technological dimensions and, accordingly, allowances, for the hypothetical workpiece with wide dimensional tolerances the technological dimensions and allowances are determined once, while it is assumed that the specific workpiece must engage with the tolerances of the hypothetical workpiece. The article deals with an example of calculating linear technological dimensions with adaptation, based on the results of monitoring and performing a separate calculation of the dimensions and allowances for each concrete workpiece in each operation. The calculation is carried out using a processing scheme, a graph, equations of dimensional chains and a computer program. As a result of the study, it was found that the calculation of linear technological dimensions with adaptation makes it possible to determine the actual values of allowances at each transition and thereby ensure predictable processing conditions.
\end{abstract}

\section{Introduction}

Technological dimensional calculations are a necessary stage of engineering process and determine the quality and economy of manufacturing. At present, when processing hard-totreat materials on NC machines, it is necessary to ensure optimal processing conditions for each workpiece based on reliable information on allowances at each step. However, according to the existing methodology for calculation of technological dimensions and, accordingly, allowances, for the hypothetical workpiece with wide dimensional tolerances the technological dimensions and allowances are determined once, while it is assumed that the specific workpiece must engage with the tolerances of the hypothetical workpiece. This means that the cutting process conditions for each specific workpiece in each operation will be unknown, unpredictable and significantly different for various workpieces, which leads to an inefficiency in the cutting process. Consequently, there is a contradiction between the

\footnotetext{
*Corresponding author: masaginvb@mail.ru
} 
possibility of providing optimal processing conditions based on scientific methods in the field of cutting theory and real processing conditions that are unpredictable due to the underdevelopment of scientific methods for calculating technological dimensions and allowances. It is required to develop a new methodology for calculation of technological dimensions and allowances with adaptation, which will allow to provide predictable and optimal processing conditions, based on monitoring and executing the separate calculation of dimensions and allowances for each particular workpiece, at each operation. This will increase the economy by reducing the consumption of tools that previously worked in suboptimal conditions and will also improve the quality of the parts produced due to greater uniformity and predictability of processing conditions.

Analysis of the existing methods for assigning tolerances and allowances for workpiece, as well as optimization methods [1-6] showed that the issue of the correct designation of tolerances and allowances for technological dimensions is very important, relevant and covers many aspects of the problem - methods for obtaining surfaces with the necessary accuracy parameters and quality, increase in efficiency, methods of assigning technological dimensions, etc. These methods can be described as the base to the solution of the problem, which relates to the variety of operative factors and their reciprocal action.

Accordingly, the existing methods of technological dimensional calculations are based on the initial data on the dimensional structure of the part and the technological process. The dimensions of the workpiece are obtained during the calculation. Design of the workpiece is carried out in accordance with the standards for the part drawing immediately, without information about the technological process. The size of tolerances for the workpiece is determined by the method of its manufacture, according to the relevant standards. Existing methods, except for the methodology [6], do not take information about the workpiece as the initial data.

\section{Description of the study}

Consider an example of the calculation of linear technological dimensions with adaptation. It is necessary to make a part (Fig. 1a) with linear design dimensions. These dimensions are known: $S_{1}=25_{-0.1} \mathrm{MM}, S_{2}=50_{-0.4} \mathrm{MM}$. The workpiece sketch and the technological sketch with linear technological dimensions are shown in Fig. 1b and Fig. 1c. Tolerance values of operational technological dimensions and minimum allowance values in all operations are determined by the reference documents. 


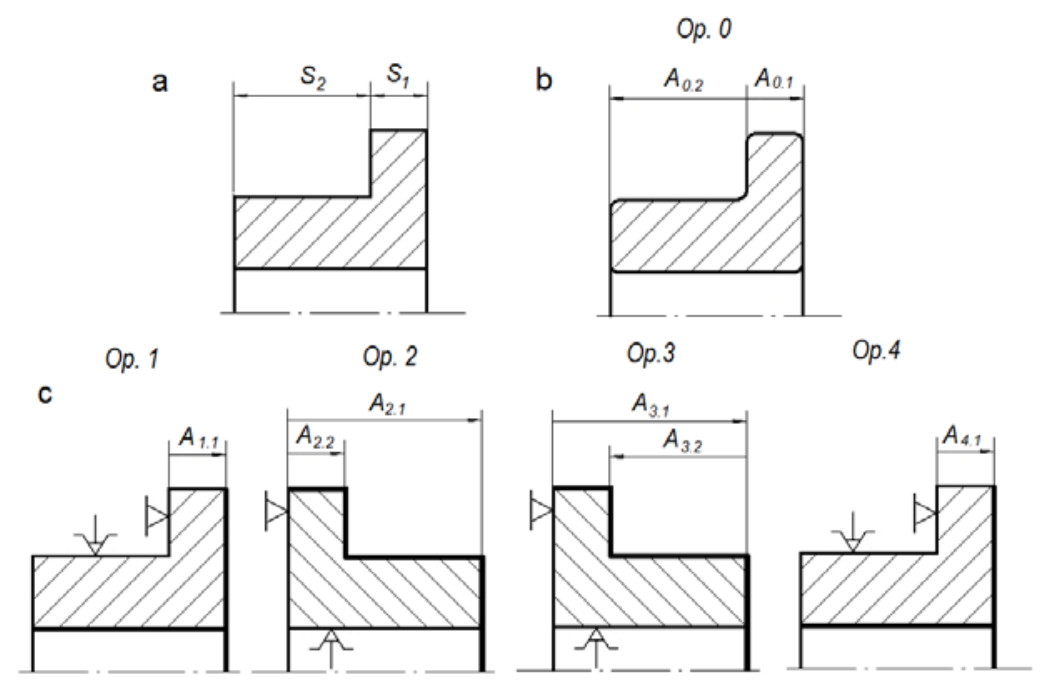

Fig. 1. Sketch of: part (a), workpiece (b), operation (c).

The methodology includes the following items that are performed before each operation.

1. Control of dimensional and precision parameters of the workpiece. Before the first operation, this is the initial workpiece, before the second and subsequent operations it is a partially processed part.

2. Determination the parameters of total machining allowances.

3. Distribution of the total allowances for operational allowances.

4. Determination of the operational tolerances providing the inaccuracy values in total allowances.

5. Calculation on the computer program of all technological dimensions and allowances for the operational allowances and tolerances specified in item 3 and 4.

6 . Verification by the computer program to ensure minimum value of operational allowances.

7. Machining of the part in accordance with the calculated dimensions and allowances.

Thus, the calculation is repeated as many times as the operations contained in the technological process. Therefore, it is required to construct an appropriate number of dimensional schemes (Fig. 2). On the graph (Fig. 3a) shows its parts corresponding to the configurations before each operation. In addition to the technological dimensions the measured dimensions before the operations that do not coincide with the technological dimensions - this is the dimensions $A_{\kappa 2}$ and $A_{\kappa 3}$ (Fig. 2b, 2d, 3a). 



Fig. 2. Part processing scheme: before the first operation (a), before the second operation (b), before the third operation (c), before the fourth operation (d).

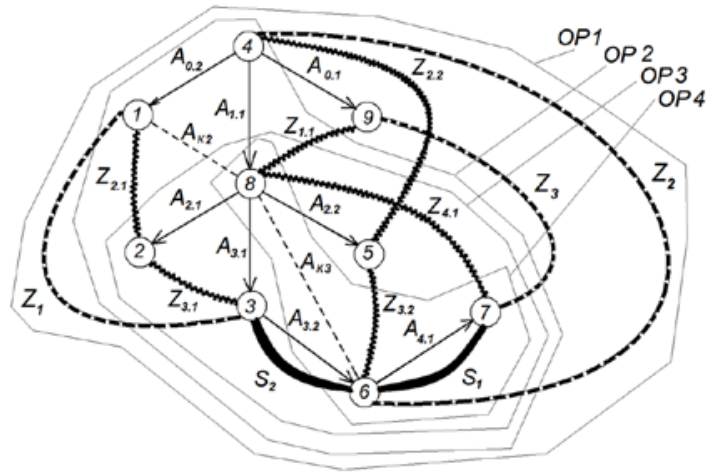

a)

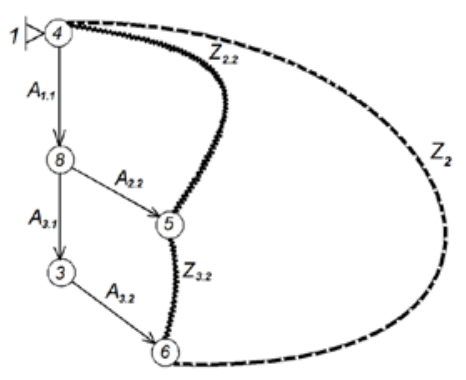

b)

Fig. 3. The graph of the technological dimensional chains (a) and the graph of the technological dimensional chains of total allowance in the first operation (b).

Performing a detailed calculation of the technological dimensions and allowances before the first operation. For the rest operations, we will immediately present the calculation results, since the calculation itself is similar.

1. Control of dimensional and precision parameters of the workpiece. 
Let the measurement results be the following: $A_{0.1}=32.4 \pm 0.1 \mathrm{~mm}, A_{0.2}=50 \pm 0.1 \mathrm{~mm}$.

2. Determination the parameters of total machining allowances.

On the graph (Fig. 3a) new edges are introduced - total machining allowances $Z_{1}, Z_{2}$, $Z_{3}$, which are shown with thick dashed lines. Then, dimensional chains are detected along the graph and equations of dimensional chains are formed, which include total allowances $Z_{i}$, the dimensions of the workpiece $A_{0 . i}$, and the design dimensions $S_{i}$. The calculation is carried out on the average sizes, two systems of equations are made. The first system of equations will include the average dimension values - the total allowances $Z_{i m}$, the workpiece dimensions $A_{0 . i m}$, and the design dimensions $S_{i m}$. In the second - the inaccuracies of the total allowances $\delta Z_{i}$, the tolerances for the workpiece dimensions $\delta A_{0 . i}$ and the tolerances for the design dimensions $\delta S_{i}$. The first equation system is composed in such a way that each of the equations includes the same total allowance, the external surface of which is the base on this operation $-Z_{2 m}$.

$$
\begin{aligned}
& Z_{1 m}+S_{2 m}-Z_{2 m}-A_{0.2 m}=0, Z_{3 m}-A_{0.1 m}+Z_{2 m}+S_{1 m}=0, \\
& \delta Z_{1}=\delta S_{2}+\delta Z_{2}+\delta A_{0.2}, \delta Z_{3}=\delta A_{0.2}+\delta Z_{2}+\delta S_{1},
\end{aligned}
$$

However, the number of unknowns - in threes - in the received systems $\left(Z_{1 m}, Z_{2 m}, Z_{3 m}\right.$ for the first system and $\delta Z_{1}, \delta Z_{2}, \delta Z_{3}$ - for the second one) is more than the number of equations, therefore for each system there is an infinite number of solutions. To obtain a single solution, we reduce the number of unknowns by preliminary determining the parameters of one of the total allowances $\left(Z_{2 m}, \delta Z_{2}\right)$ present in all corresponding equations.

The external surface of the total allowance $Z_{2}$ is the base on the first operation. The total allowance $Z_{2}$ consists of two operational allowances $Z_{2.2}$ and $Z_{3.2}$ (Fig. 3b). For the total allowance, determined its minimum required value $\left(Z_{\min 2}\right)$, is equal to the sum of the minimum required operational allowances $\left(Z_{\min 2.2}\right.$ and $\left.Z_{\min 3.2}\right)$, and an inaccuracy $\left(\delta Z_{2}\right)$ equal to the sum of the expected inaccuracies $\left(\delta Z_{2.2}\right.$ and $\left.\delta Z_{3.2}\right)$ in the components of the operating allowances, therefore, its mean value and inaccuracy is determined. Let the minimum required operational allowances be the following: $0.5 \mathrm{~mm}$ for the first treatment, $0.2 \mathrm{~mm}$ - for the second). Therefore, $Z_{\min 2}=Z_{\min 1}=Z_{\min 3}=0.7 \mathrm{~mm}$. Using the technological tolerances for dimensions $\left(\delta A_{1.1}=0.71, \delta A_{2.2}=0.21, \delta A_{3.1}=0.12, \delta A_{3.2}=0.4\right)$, determine determine inaccuracies of the total allowance $\delta Z_{2}$ on the initial base: $\delta Z_{2}=1.65 \mathrm{~mm}$.

After that, we determine by the equations (2) and the values of design tolerances and tolerances for the workpiece dimensions $\left(\delta S_{1}=0.1 \mathrm{~mm}, \delta S_{2}=0.4 \mathrm{~mm}, \delta A_{0.1}=0.2 \mathrm{~mm}\right.$, $\delta A_{0.2}=0.2 \mathrm{~mm}$.) the inaccuracies of the remaining total allowances: $\delta Z_{1}=2.25 \mathrm{~mm}, \delta Z_{3}=$ $1.95 \mathrm{~mm}$.

Determine the average total allowance $Z_{2 m}$ on the initial base by the minimum required total allowances $Z_{\min 2}$ and the tolerance of the total allowance $\delta Z_{2}: Z_{2 m}=1.525 \mathrm{~mm}$. Then, using equations (1), we determine the average values of the remaining total allowances: $Z_{1 m}=1.725 \mathrm{~mm}, Z_{3 m}=5.925 \mathrm{~mm}$.

Determine the real values of the minimum total allowances and perform the verification of the received real minimum values of the total allowances for compliance with the requirement $Z_{\text {dmin } i} \geq Z_{\min i}$. The obtained value of the allowance $Z_{d \min 1}=0.6 \mathrm{~mm}$ does not satisfy the requirement $Z_{d \min i} \geq Z_{\text {mini }}$. To ensure the minimum required value of this allowance $Z_{\min 1}=0.7 \mathrm{~mm}$, it is required to increase the obtained value $Z_{d \min 13}=0.6 \mathrm{~mm}$ by $\Delta Z=0,1 \mathrm{~mm}$ due to the increase of the total minimum allowance on the surface - the initial base to the value $Z_{d \min 2}=0,8 \mathrm{~mm}$. It is more advisable to equalize the minimum allowances. The magnification of $Z_{d \min 2}$ will be $2.125 \mathrm{~mm}$. Then: $Z_{d \min 1}=2.725 \mathrm{~mm}, Z_{d \min 2}$ $=2.825 \mathrm{~mm}, Z_{d \min 3}=2.825 \mathrm{~mm}, Z_{1 m}=3.85 \mathrm{~mm}, Z_{2 m}=3.65 \mathrm{~mm}, Z_{3 m}=3.8 \mathrm{~mm}$.

3. Distribution of the total allowances for operational allowances. 
Based on the obtained real values of the minimum total allowances, by distributing in accordance with the coefficient $K_{i}$, which considers the dependence of the amount remove allowance on the side of the number of treatments, we calculate the real values of the minimum operational allowances that provide the values of the real minimum total allowances (Table 1).

Table 1. Determination of real minimum operatiomal allowances.

\begin{tabular}{|c|c|c|c|c|}
\hline Index & Value, $\boldsymbol{m m}$ & Index & Coefficient $\boldsymbol{K}_{\boldsymbol{i}}$ & Value, $\boldsymbol{m m}$ \\
\hline \multirow{2}{*}{$Z_{d \min 1}$} & \multirow{2}{*}{2.725} & $Z_{\text {dmin } 2.1}$ & $5 / 7$ & 1.95 \\
\cline { 3 - 5 } & \multirow{2}{*}{$Z_{d \min 2} 2.825$} & $Z_{\text {dmin } 3.1}$ & $2 / 7$ & 0.78 \\
\cline { 3 - 5 } & \multirow{2}{*}{$Z_{\text {dmin } 3}$} & $Z_{\text {dmin } 2.2}$ & $5 / 7$ & 2.02 \\
\cline { 3 - 5 } & \multirow{2}{*}{2.825} & $Z_{\text {dmin } 3.2}$ & $2 / 7$ & 0.81 \\
\cline { 3 - 5 } & & $Z_{\text {dmin } 1.1}$ & $5 / 7$ & 2.02 \\
\hline
\end{tabular}

4. Determination of the operational tolerances providing the inaccuracy values in total allowances.

Preliminarily, according to reference data, technological tolerances for all technological dimensions are specified, excluding the previously assigned tolerances: $\delta A_{2.1}=0.3 \mathrm{~mm}$, $\delta A_{4.1}=0.1 \mathrm{~mm}$. With the help of the computer program, the expected inaccuracy in the total allowances $\rho Z_{i}$ at the assigned technological tolerances are determined: $\rho Z_{1}=\rho Z_{2.1}+\rho Z_{3.1}$ $=1,63 \mathrm{~mm}, \rho Z_{3}=\rho Z_{1.1}+\rho Z_{1.4}=1,53 \mathrm{~mm}$.

Required values: $\delta Z_{1}=2.25 \mathrm{~mm}, \delta Z_{3}=1.95 \mathrm{~mm}$. It is necessary to expand the expected inaccuracies of total allowances: $\rho Z_{1}$ by $0.62 \mathrm{~mm}, \rho Z_{3}$ by $0.42 \mathrm{~mm}$. It is possible to expand $\rho Z_{1}$ by increasing $\delta A_{2.1}$ by $0.31 \mathrm{~mm}$, since $\delta A_{2.1}$ enters in the $\rho Z_{1}$ twice. Expanding $\rho Z_{3}$ could be due to an increase in $\delta A_{4.1}$ by $0.42 \mathrm{~mm}$, but with an increase in $\delta A_{4.1}$, the design tolerance $\delta S_{1}$ will not be provided. Therefore, we will compensate the difference between $\rho Z_{3}$ and $\delta Z_{3}$ by expanding the minimum allowance $Z_{d m i n}$. We form the equation for calculating a new value $Z_{d \min 3 u}: Z_{d \min 3 u}=Z_{3 m}+0,5 \cdot\left(\delta Z_{3}-\rho Z_{3}\right)=4.01 \mathrm{~mm}$. In this case, the minimum allowances will change: $Z_{d \min 1.1 u}=2.17 \mathrm{~mm}$ and $Z_{d \min 4.1 u}=0.87 \mathrm{~mm}$.

5. Calculation of technological dimensions, including the workpiece dimensions.

It is carried out by means of the computer program.

6. Verification of ensuring minimum operational allowances.

When ensuring the minimum operational allowances, the settlement stage is completed, otherwise the real minimum total allowances and the real minimum operational allowances are redistributed for increasing the unrealized real minimum operational allowances.

Similarly, the calculation of the technological dimensions and allowances before the rest of the operations is performed. The results of the calculation for all operations are presented in Table 2 and Table 3.

Table 2. Linear technological dimensions.

\begin{tabular}{|c|c|c|c|c|}
\hline Index & Before 1 op. & Before 2 op. & Before 3 op. & Before 4 op. \\
\hline$A_{0.1}$ & $32.4 \pm 0.1$ & & & \\
\hline$A_{0.2}$ & $50.0 \pm 0.1$ & & & \\
\hline$A_{\mathrm{K} 2}$ & & $79.5 \pm 0.1$ & & \\
\hline$A_{1.1}$ & $29.75 \pm 0.355$ & $29.5 \pm 0.1$ & & \\
\hline$A_{2.1}$ & $77.15 \pm 0.305$ & $77.35 \pm 0.25$ & $77.2 \pm 0.05$ & \\
\hline$A_{2.2}$ & $27.30 \pm 0.105$ & $27.55 \pm 0.105$ & $26.6 \pm 0.05$ & \\
\hline$A_{3.1}$ & $75.95 \pm 0.060$ & $76.25 \pm 0.060$ & $76.1 \pm 0.060$ & \\
\hline$A_{3.2}$ & $49.8 \pm 0.2$ & $49.8 \pm 0.2$ & $49.8 \pm 0.2$ & $49.8 \pm 0.2$ \\
\hline$A_{\mathrm{K} 3}$ & & & & $26.3 \pm 0.05$ \\
\hline$A_{4.1}$ & $24.95 \pm 0.05$ & $24.95 \pm 0.05$ & $24.95 \pm 0.05$ & $24.95 \pm 0.05$ \\
\hline
\end{tabular}


Table 3. Operational allowances.

\begin{tabular}{|c|c|c|c|c|}
\hline Index & Before 1 op. & Before 2 op. & Before 3 op. & Before 4 op. \\
\hline$Z_{1.1}$ & $2.195+0.91$ & & & \\
\hline$Z_{2.1}$ & $1.84+1.52$ & $1.8+0.7$ & & \\
\hline$Z_{2.2}$ & $2.99+0.92$ & $1.745+0.41$ & & \\
\hline$Z_{3.1}$ & $0.835+0.73$ & $0.79+0.62$ & $0.99+0.22$ & \\
\hline$Z_{3.2}$ & $0.785+0.73$ & $0.735+0.73$ & $0.99+0.62$ & \\
\hline$Z_{4.1}$ & $0.89+0.62$ & $1.19+0.62$ & $1.04+0.62$ & $1.25+0.2$ \\
\hline
\end{tabular}

Thus, gradually, as far as processing, according to the results of the control of the received dimensions; technological dimensions, tolerances and allowances are defined more accurately. As can be seen from Table 3 the values of allowances before subsequent operations may differ by $30-40 \%$ in relation to those calculated in the previous stage.

\section{Conclusion}

As a result of the study, it was found that the calculation of linear technological dimensions with adaptation makes it possible to determine the actual values of allowances at each transition and thereby provide predictable processing conditions, which will reduce the cost of the tool and improve the quality of the parts produced from hard-to-treat materials. Within certain limits, due to the redistribution of allowances, the developed methodology ensures correction of the defect, resulting from the exit the workpiece dimensions beyond the established limits after processing.

\section{References}

1. B.K.A. Ngoi, Int. J. Adv. Manuf. Technol., 7, 187-192, (1992).

2. P. Ji, Int. J. of Adv. Manuf. Technol., 9, 362-368, (1994).

3. P. Ji, J. B. Xue, Int. J. of Adv. Manuf. Technol., 20, 649-654, (2002).

4. W. Bouzid Sai, W Dhifalli, A. Zghal, Int. J. Adv. Manuf. Technol., 31, 71-77, (2006).

5. P. Ji, J. B. Xue, Proc. of the Inst. of Mechanical Engineers, 220, 883-892, (2006).

6. V.A. Bartholomew, V.B. Masyagin, Omsk Scientific Bulletin, 1, 41-45, (2011). 